\title{
Enhancing Gabor Wavelets Using Volumetric Fractal Dimension
}

\author{
Alvaro Gomez Zuniga ${ }^{1}$ and Odemir Martinez Bruno ${ }^{2}$ \\ 1 Instituto de Ciências Matemáticas e de Computação \\ 2 Instituto de Física de São Carlos \\ Universidade de São Paulo \\ Av. Trabalhador São Carlense 400, São Carlos, São Paulo Brasil \\ alvarog@icmc.usp.br \\ bruno@ifsc.usp.br
}

\begin{abstract}
Texture plays an important role on image analysis and computer vision. Local spatial variations of intensity and color indicate significant differences among several types of surfaces. One of the most widely adopted algorithms for texture analysis is the Gabor wavelets. This technique provides a multi-scale and multi-orientation representation of an image which is capable of characterizing different patterns of texture effectively. However, the texture descriptors used does not take full advantage of the richness of detail from the Gabor images generated in this process. In this paper, we propose a new method for extracting features of the Gabor wavelets space using volumetric fractal dimension. The results obtained in experimentation demonstrate that this method outperforms earlier proposed methods for Gabor space feature extraction and creates a more accurate and reliable method for texture analysis and classification.
\end{abstract}

Keywords: Volumetric fractal dimension; Texture analysis; Gabor Wavelets; Feature extraction.

\section{Introduction}

Texture reflects the variations of optical properties on object surfaces. Different surfaces produce distinctive texture patterns. This makes texture an important source of discriminating information for image classification [1. Recently Gabor filters have become a widely used technique for texture analysis. These filters have desirable characteristics of localization on spatial and frequency domains being the best method to represent a signal jointly in both domains. Using this filter we can create multi-scale and multi-orientation representations of an image each with unique characteristics. The process consists on the convolution of an image with a series of filters constructed by varying its scales and orientations. Energy is then used as the descriptor of each convoluted image to form the final feature vector. However, a single measure such as energy has a limited power in seizing the richness of detail of the generated images. Recent work done on

I. Bloch and R.M. Cesar, Jr. (Eds.): CIARP 2010, LNCS 6419, pp. 362 369, 2010.

(C) Springer-Verlag Berlin Heidelberg 2010 
the field attempts to generate methods to extract more useful information of the Gabor space: First and second order statistic descriptors. Invariant moments that achieve resistance to rotation, translation and scaling [8] 9, 4, [1]. And more recently, the local binary pattern (LBP) operator offering the best results found in the literature [12, 13, , 14.

In this paper, we introduce the use of fractal descriptors on the Gabor space as an alternative to improve the feature extraction process. This proposed method improves the results obtained by the Gabor wavelets process. This new method consists in calculating the fractal signature of each convoluted image and generating a feature vector that concatenates these signatures into a final vector.The article starts by making a quick review of Gabor filters and feature extraction from Gabor space. Next, the proposed method is presented. Finally experiments are performed with the Brodatz texture database where the method is compared with the most important feature extraction methods of the Gabor space found on the literature: first and second order statistics and local binary patterns. The article ends with the final discussions and conclusions.

\section{Gabor Wavelets}

In recent years the Gabor filter has become popular in image processing and analysis, particularly in texture feature extraction [3]. Besides the classical approach in texture, the filter has been used in other applications such as biometrics [23], image segmentation [8], pattern recognition and OCR 24].

The two-dimensional Gabor transform is a complex sine wave with frequency $W$ modulated by a Gaussian function. Its form in space $g(x, y)$ and frequency domains $G(u, v)$, is given by Eqs 1 and 2 ,

$$
\begin{gathered}
g(x, y)=\left(\frac{1}{2 \pi \sigma_{x} \sigma_{y}}\right) \exp \left[-\frac{1}{2}\left(\frac{x^{2}}{\sigma_{x}^{2}}+\frac{y^{2}}{\sigma_{y}^{2}}\right)+2 \pi j W x\right] \\
G(u, v)=\exp \left\{-\frac{1}{2}\left[\frac{(u-W)^{2}}{\sigma_{u}^{2}}+\frac{v^{2}}{\sigma_{v}^{2}}\right]\right\}
\end{gathered}
$$

Although the Gabor transforms is older than wavelets, due to its multi-scale nature it can be used as mother wavelet. For the process is employed a filter bank constructed by varying the scale and orientations of each filter. In [3] the authors describe how to compose the bank of Gabor filters to make the Gabor wavelet with a set of parameters that assures maximum spectrum coverage with lowest redundancy. We use this approach to design the Gabor wavelet.

The Most common approach to analyze and characterize textures with Gabor is to adopt a set of filters with different scales and orientations. This characterizes the Gabor wavelets process [3], then each filter is convoluted with the image as shown in Figure 1, For each of the spaces obtained a feature is extracted. A simple way of extracting this feature is using energy as the general descriptor. The energy of each space is concatenated into a feature vector according to: 


$$
f=\left[E_{11}, E_{12}, \ldots, E_{21}, E_{22}, \ldots, E_{M N}\right]
$$

Where $M$ and $N$ are the number of scales and orientations respectively.

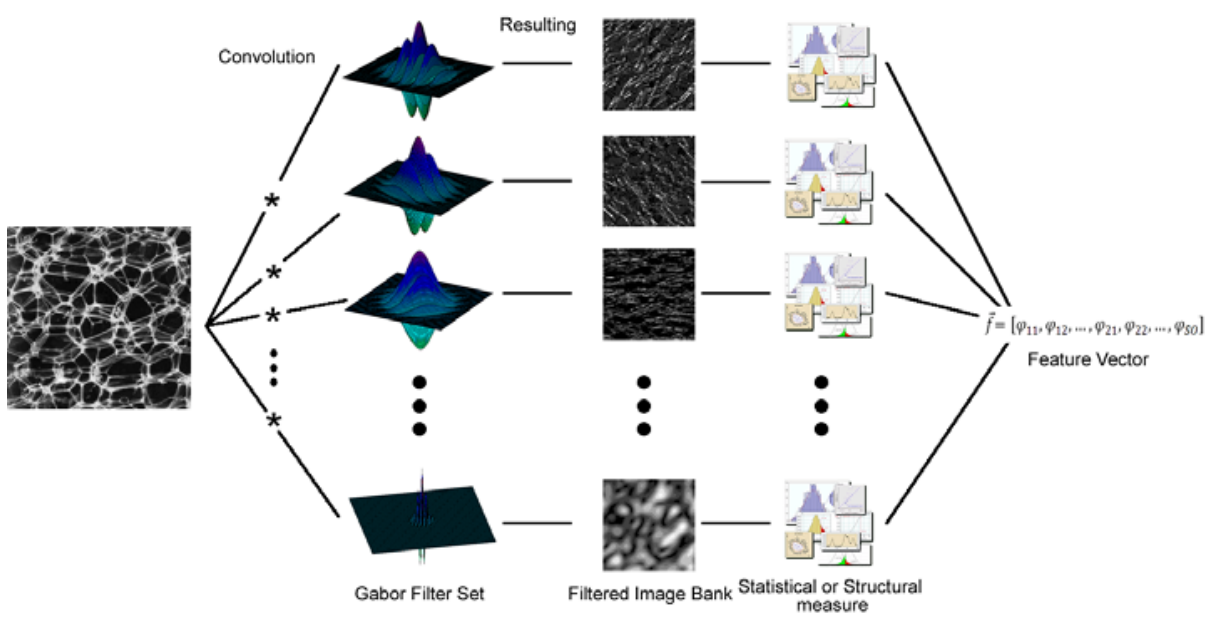

Fig. 1. General process used to obtain the Gabor signatures

\section{$3 \quad$ Feature Extraction on the Gabor Space}

Most of the methods found in the literature makes use of statistical descriptors. Being fairly simple to compute, they have the advantage of generating feature vectors of low dimensionality. The descriptors that obtain the best results are based on first and second order statistics: Variance, energy and percentiles [5]. Other approaches use descriptors based on the local binary pattern (LBP) operator. The LBP operator analyzes the image using a nxn window generating a binary code for each region. Then a histogram with the values obtained for each window is generated and this represents the feature vector of the image. The LBP operator has proven to be one of the most suitable texture descriptors found on the literature, and it has been successfully applied in the Gabor wavelets process [12] (LBP1), 13] (LBP2).

\section{Volumetric Fractal Dimension}

The fractal concept was first used by Mandelbrot in his book [18. This concept suggests that natural objects are not formed by Euclidean geometry objects; instead, they form sub-patterns that are repeated to form more complex objects. In recent years this concept has been widely used in the field of image analysis [19], 20]. Many natural forms have fractal patterns (structures of plants, coastlines, plants). The fractal dimension of the image is taken as a measure of the irregularity of the objects. However, in order to calculate the fractal dimension 
(FD) of non-fractal objects we need a method to estimate the FD in discrete finite images. One of the most accurate methods to calculate the fractal dimension of a non-fractal object is the Bouligand-Minkowski method 19. To calculate the volumetric fractal dimension (VFD) using the Minkowski-Bouligand method on a grayscale image we must first readapt the image. For this we use the approach taken in 20]. The 3-D projection of the image is generated from a grayscale image to meet the conditions to apply the Euclidean distance transform. Each pixel of the image is transformed into a point $p=(y, x, z) \epsilon S$, where $Y$ and $X$ correspond to the coordinates of the pixel in the image and $z=f(x, y)$ is the intensity. Then the Minkowski-Bouligand fractal dimension of the surface $S$ can be estimated by:

$$
\begin{gathered}
F D=3-\lim _{r \rightarrow>0} \frac{\log (V(r))}{\log (r)} \\
V(r)=\left\{p^{\prime} \epsilon R^{3}|\exists p \epsilon S:| p-p^{\prime} \mid \leq r\right\}
\end{gathered}
$$

This method calculates the variation of a volume $V(r)$ given by the application of exact dilations of the image $f(x, y)$ with a sphere of radius $r$. Small changes on the structure of the image can produce significant changes in the calculated FD. The FD and $V(r)$ are given by:

$$
\begin{gathered}
E=1, \sqrt{2}, \sqrt{3}, \ldots, r_{\max } \\
\psi\left(r_{\max }\right)=\left[\log V(1), \log V(\sqrt{2}), \log V(\sqrt{3}), \ldots, \log V\left(r_{\max }\right)\right]
\end{gathered}
$$

\section{Experimentation and Evaluation}

Image database. In experiments we used the Brodatz texture database [22]. This album has been widely used to test and compare techniques used to extract texture features. The album is composed of 111 images of artificial textures. From this album we derive an image database formed by selecting 10 random non-overlapping windows of $200 \times 200$ pixels size from each image in the original album. These images are coded in 8-bit depth and saved in a lossless format. The final database generated is composed of 1110 images with 111 classes and 10 images per class. Some samples are shown in Figure 2

The classification of the samples was made using a Bayesian probability method. Specifically, the Naïve Bayes method. The 1110 samples are divided in training and testing set in a proportion of 8 to 2 . This means $80 \%$ of the samples were used for training and $20 \%$ for testing with a random selection of each set ensuring that each class is represented with a consistent number of samples. This scenario is repeated 10 times in order to obtain an accurate and robust estimation of the predictions for each class. Moreover, to determinate the best combination of the Gabor parameters and the feature extractor we use a wide range of scales and orientations for all the experiments. The near ideal parameters for the Gabor filters are extracted from [3]. 

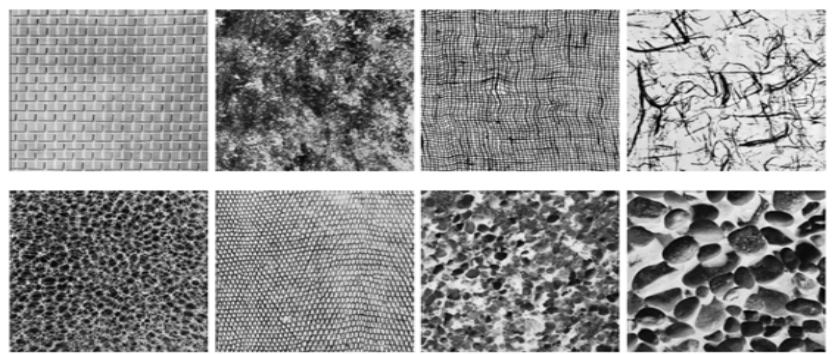

Fig. 2. Sample images from the Brodatz texture database used in experimentation

Table 1. Results for experimentation using the feature extractors explained

\begin{tabular}{cccccc}
\hline $\begin{array}{c}\text { No of } \\
\text { Descriptors }\end{array}$ & $\begin{array}{c}\text { Average } \\
\text { No. Of } \\
\text { Correctly } \\
\text { Classified } \\
\text { Images }\end{array}$ & $\begin{array}{c}\text { Average } \\
\text { Success } \\
\text { Rate (\%) }\end{array}$ & $\begin{array}{c}\text { Standard } \\
\text { Deviation }\end{array}$ & $\begin{array}{c}\text { Root } \\
\text { Relative } \\
\text { Squared } \\
\text { error(\%) }\end{array}$ & Reliability \\
\hline 1 & 185.26 & 83.45 & 2.78 & 63.08 & \\
3 & 191.12 & 86.09 & 1.8 & 52.67 & 86.32 \\
7 & 192.67 & 86.79 & 2.09 & 50.48 & 87.24 \\
11 & 200.67 & 90.39 & 2.59 & 40.51 & 90.42 \\
16 & 201.11 & 90.59 & 1.99 & 39.38 & 91.79 \\
20 & 201.44 & 90.74 & 2.12 & 41.82 & 92.71 \\
22 & 204 & $\mathbf{9 1 . 8 9}$ & 1.65 & 38.8 & 93.62 \\
30 & 200.55 & 90.34 & 1.91 & 44.59 & 91.5 \\
38 & 196 & 88.29 & 2.31 & 48.32 & 89.06 \\
46 & 194.67 & 87.69 & 3.03 & 50.28 & 86.33 \\
\hline
\end{tabular}

The use of Eq. 7 generates a signature $\psi$ composed of $N$ descriptors where $N$ is the number of exact dilatations possible for a radius $r_{\max }$. In order to reduce dimensionality we used a technique to extract the most important features of the signature called Fourier descriptors. Using this technique we obtain the approximate number of descriptors necessary to obtain the best results while keeping the dimensionality of the feature vector as low as possible.

The average success rate, standard deviation (of the success rate), the root relative squared error and reliability (average a posteriori probability for samples correctly classified) are presented in the Table 1. These values are used to asses the results and accuracy of the data modeled using the proposed technique. The maximum radius of expansion used for Eq. 7 was 9 and 16. According to [20] these values obtain the best results in classification. We only present the table corresponding to the results using a radius of 16 because it was proven on [20] that these value achieves better performance and more reliable results. 
Table 2. Results for experimentation with the feature extractors applied to the Gabor space

\begin{tabular}{cccccccccc}
\hline \multicolumn{10}{c}{ Scales x Orientations } \\
\hline Gabor + & $\mathbf{2} \times \mathbf{6}$ & $\mathbf{3} \times \mathbf{4}$ & $\mathbf{3} \times \mathbf{5}$ & $\mathbf{4} \times \mathbf{4}$ & $\mathbf{4} \times \mathbf{6}$ & $\mathbf{5} \times \mathbf{5}$ & $\mathbf{6} \times \mathbf{3}$ & $\mathbf{6} \times \mathbf{6}$ \\
\hline VFD & 89.06 & 90.64 & 90.32 & 90.84 & $\mathbf{9 1 . 8 9}$ & 91.02 & 89.46 & 88.22 \\
Energy & 60.81 & 80.43 & 79.13 & 79.83 & $\mathbf{8 1 . 9 3}$ & 81.78 & 76.83 & 81.83 \\
Variance & 64.76 & 82.13 & $\mathbf{8 3 . 7 3}$ & 79.98 & 82.68 & 81.03 & 77.28 & 82.38 \\
Percentil25 & 61.36 & 76.88 & 75.07 & 79.48 & 81.18 & $\mathbf{8 2 . 7 3}$ & 77.93 & 80.58 \\
Percentil75 & 62.71 & 80.98 & 80.78 & $\mathbf{8 3 . 0 3}$ & 83.08 & 82.93 & 79.23 & 82.23 \\
LBP1 & 86.58 & 87.02 & 88.14 & $\mathbf{8 8 . 9 9}$ & 85.54 & 83.03 & 87.39 & 76.53 \\
LBP2 & 87.04 & 85.79 & $\mathbf{8 8 . 2 9}$ & 87.29 & 86.54 & 84.63 & 86.29 & 84.63 \\
\hline
\end{tabular}

Table 1 shows that the best result is obtained with 22 descriptors of the fractal signature obtaining a mean average success of $91.89 \%$ using a radius of 16 for Eq. 7 for the proposed technique. The results obtained can be compared with the other feature extractors in Table 2 using several parameters for the Gabor Wavelets. In experimentation we determinate that the alteration of other parameters on the Gabor process produces similar variations on all the methods without any significantly improve or deprecation on the results; therefore, we only used $U_{l}=0.05, U_{h}=0.3$ as fix parameters for the Gabor Wavelets.

In our experiments energy feature achieves $81.93 \%$ of images correctly classified on 4 scales and 6 orientations in concordance with the model and results presented on [3]. For the other feature extractors results are increasingly better accordingly to the technique used obtaining close results with the LBP methods but with higher dimensionality. From the results observed it can be shown that the proposed method offers the best performance and presents a great alternative for improving the Gabor wavelets process.

\section{Conclusions}

This paper presents a new approach for feature extraction by applying volumetric fractal dimension instead of energy in the Gabor wavelets process. The proposed method obtains significantly better results than other methods studied on the literature. Both the accuracy and reliability of the model show its robustness and reliability. The proposed method achieved $91.89 \%$ of images correctly classified with $93.62 \%$ reliability while maintaining an acceptable dimensionality. Therefore, considering the promising results in texture analysis and classification we can determine that the volumetric fractal dimension is a suitable technique to exploit the richness of detail of the images resulting from the convolution on the Gabor wavelets process. The method can be applied in many classification tasks due to the power of effectively representing and differentiating micro and macro texture. 


\section{Acknowledgment}

A. G. Z. gratefully acknowledges the financial support of FAPESP (The State of Sao Paulo Research Foundation) Proc. 2009/04362. O.M.B. acknowledges support from CNPq (306628/2007-4).

\section{References}

1. Rosenfeld, A., Lipkin, B.S.: Picture Processing and Psychopictorics. Academic Press, London (1970)

2. Daugman, J.G.: Two-dimensional spectral analysis of cortical receptive field profiles. Vision Research 20, 847-856 (1980)

3. Manjunath, B.S., Ma, W.-Y.: Texture features for browsing and retrieval of image data. IEEE PAMI, 837-842 (1996)

4. Andrysiak, T., Choras, M.: Image Retrieval Based on Hierarchical Gabor Filters. Intl J. Applied Mathematics and Computer Science 15(4), 471-480 (2005)

5. Bandzi, P., Oravec, M., Pavlovicova, J.: New Statistics for Texture Classification Based on Gabor Filters. Intl. Radioengineering J. 16(3), 133-137 (2007)

6. Clausi, D.A., Deng, H.: Fusion of Gabor filter and co-occurrence probability features for texture recognition. IEEE Transactions on Image Processing 14(7), 925$936(2005)$

7. Shahabi, F., Rahmati, M.: Comparison of gabor-based features for writer identification of farsi/arabic handwriting. In: Proc. of 10th Intl. Workshop on Frontiers in Handwriting Recognition, pp. 545-550 (2006)

8. Muneeswaran, K., Ganesan, I., Arumugam, S., Harinarayan, P.: A novel approach combining gabor wavelet and moments for texture segmentation. Intl. J. of Wavelets, Multiresolution and Information Processing 3(4), 559-572 (2005)

9. Qaiser, N., Hussain, M., Hussain, A., Qaiser, N.: Texture Recognition by Fusion of Optimized Moment Based and Gabor Energy Features. Intl. J. CSNS 8(2), 264-270 (2008)

10. Andrysiak, T., Choras, M.: Image Retrieval Based on Hierarchical Gabor Filters. Intl. J. Applied Mathematics and Computer Science 15(4), 471-480 (2005)

11. Grigorescu, S.E., Petkov, N., Kruizinga, P.: Comparison of texture Features based on Gabor Filters. IEEE Transactions on Image processing 11(10), 1160-1167 (2002)

12. Zhang, W., Shan, S., Gao, W., Chen, X., Zhang, H.: Local Gabor Binary Pattern Histogram Sequence (LGBPHS): A Novel Non-Statistical Model for Face Representation and Recognition. In: Tenth IEEE ICCV, vol. 1, pp. 786-791 (2005)

13. Lei, Z., Liao, S., He, R., Pietikäinen, M., Li, S.: Gabor volume based local binary pattern for face representation and recognition. In: 8th IEEE Intl. Conference on Automatic Face and Gesture Recognition, pp. 1-6 (2008)

14. Ojala, T., Pietikäinen, M., Mäenpää, T.: Multiresolution Gray-Scale and Rotation Invariant Texture Classification with Local Binary Patterns. IEEE Transactions on Pattern Analysis and Machine Intelligence 24(7), 971-987 (2002)

15. Casanova, D., de Mesquita Sá Jr., J.J., Bruno, O.M.: Plant leaf identification using Gabor wavelets. Intl. J. of Imaging Systems and Technology 19(3), 236-243 (2009)

16. Haralick, R.M., Shanmugam, K., Dinstein, I.: Textural Features for Image Classification. IEEE Trans. Sys. Man Cybern. 3, 610-621 (1973)

17. Qaiser, N., Hussain, M.: Optimum Window-size Computation for Moment Based Texture Segmentation. In: Proc. IEEE INMIC, pp. 25-29 (2003) 
18. Mandelbrot, B.B.: The Fractal Geometry of Nature, W.H. (1982)

19. Backes, A.R., Bruno, O.M.: Fractal and Multi-Scale Fractal Dimension analysis: a comparative study of Bouligand-Minkowski method. INFOCOMP (UFLA) 7, 74-83 (2008)

20. Backes, A.R., Casanova, D., Bruno, O.M.: Plant leaf identification based on volumetric fractal dimension. IEEE PAMI 23, 1145-1160 (2009)

21. Fabbri, R., Da Costa, F.L., Torelli, J.C., Bruno, O.M.: 2D Euclidean distance transform algorithms: A comparative survey. ACM Computing Surveys (CSUR) 40(1), $1-44$ (2008)

22. Brodatz, P.: Textures; a photographic album for artists and designers (1996)

23. Daugman, J.: How iris recognition works. IEEE Transactions on Circuits and Systems for Video Technology 14, 21-30 (2004)

24. Daugman, J.: Gabor wavelets and statistical pattern recognition. In: The Handbook of Brain Theory and N.N., 2nd edn., pp. 457-463. MIT, Cambridge (2002) 